\title{
Interdisciplinary connections in teaching mathematical and environmental disciplines to students
}

\author{
Marina Vinogradova ${ }^{1, *}$ \\ ${ }^{1}$ Ural State University of Railway Transport USURT, Tyumen, Russia
}

\begin{abstract}
This article deals with the implementation of interdisciplinary relations through the problems of applied nature of students studying in the specialty 23.05.04 Operation of railway doro on the example of the implementation of the discipline «Mathematics». The purpose of this article is to teach the student to solve problems of an applied nature that are of interest to the student. When using a subject-based learning system, interdisciplinary connections allow us to solve the existing contradictions between the disparate assimilation of knowledge and the need for their application, both holistic and complex, in the practical life of a person. As a result, knowledge becomes not only concrete, but also generalized, which gives students the opportunity to transfer this knowledge to new situations and apply it in practice. The article substantiates the need for interdisciplinary connections to reflect the integrity of nature in the content of educational material, to create a true system of knowledge and understanding of the world.
\end{abstract}

\section{Introduction}

An integral criterion for assessing the professional image of a specialist is the graduate's readiness for professional activity as a condition of his competitiveness. One of the indicators of a graduate's competitiveness is his demand in the labor market. When organizing an educational process focused on a competency-based approach, the main goal of a qualitative renewal of vocational education is to train a specialist able to consciously use the potential of fundamental disciplines for the holistic solution of professional problems. This is possible due to interdisciplinary integration that is, combining knowledge and practical actions at all stages of training.

Professional competence is the ability to successfully apply the knowledge gained, practical experience, intelligence and personal qualities in their professional activities [1]. When mastering general professional competencies, a future specialist in the field of railway operation must master the ability to apply methods of mathematical analysis and modeling, theoretical and experimental research.

Engineering education requires deep knowledge in the field of mathematics and its methods, which are fundamental for many disciplines and contribute to the development of

\footnotetext{
* Corresponding author: vinmarvlad@yandex.ru
} 
a fundamental system of training students in the process of their university education [2]. It should be noted that today this problem has been sufficiently well studied, but with the help of problems of an applied nature, we will consider the implementation of interdisciplinary connections in the study of the discipline «Mathematics».

A carefully thought-out teaching methodology for all its components is necessary for the successful functioning of the pedagogical system. Any modern pedagogical technology is a synthesis of the achievements of pedagogical science and practice, a combination of traditional elements of experience and what is generated by social progress, humanization and democratization of society.

In classical pedagogy, in particular in the works of J. A. Comensky, D. Lock, I. G. Pestalozzi, much attention was paid to the problem of interrelationships between academic subjects. They substantiated the need for interdisciplinary connections to reflect the integrity of nature in the content of educational material, to create a true system of knowledge and worldview.

So, Ya. A. Komensky noted that everything that is in mutual connection should be taught in the same connection [3].

After analyzing the psychological, pedagogical, scientific literature, we concluded that the integration of the educational process is one of the most important factors in optimizing the learning process. The need for interdisciplinary integration, which is much broader than interdisciplinary connections, stems from their pedagogical, philosophical and psychological meanings to improve the learning process.

According to such authors as Malchukova, N.N., Kryucheva, Y.V.Biryukova N.V., Yakobyuk L.I., Zenina, N.V. it is not enough to be a good specialist, you need to be a good employee, namely a person who can work in a team, will be able to make decisions independently, will be mobile [4], capable of changes in the labor market. In this case, interdisciplinary connections come to the rescue, which are reflected in the content of educational disciplines of those dialectical relations that objectively operate in nature and are known to modern science. Integration consolidates not only the relationship, but also the interpenetration of individual academic disciplines into each other. In psychology and pedagogy, the conclusion is substantiated that interdisciplinary ties are one of the important psychological and pedagogical conditions for increasing the scientific capacity and accessibility of learning, its connection with the surrounding reality, enhancing cognitive activity and improving the process of forming knowledge, skills and abilities from students [5-7].

Thus, within the framework of interdisciplinary relations, the teacher has a goal to teach the student to solve problems of an applied nature that are of interest to students, motivates him, and in which the condition is a description of a specific life situation related to the knowledge and experience of students. In connection with the above, a task is built for the teacher of higher education: to increase the volume of tasks in the applied direction of teaching mathematics, for further professional growth and competitiveness in difficult market conditions.

\section{Materials and methods}

The study was conducted on the basis of the branch of the Federal State Budgetary Educational Institution of Higher Professional Education «Ural State University of Railway Transport» in Tyumen. To conduct the experiment, two experimental groups of students were identified, 20 people in each group, studying in the specialty 23.05.04 Railway operation. The following research methods were used: analysis of psychological, pedagogical and methodological literature, as well as generalization of the content of concepts and categories; qualitative and quantitative analysis of the obtained data; methods of statistical data 
processing. Currently, the process of education in universities has undergone significant changes. They are associated with the inevitable increase in the flow of scientific information, the need to process it in a short time, the use of various technical teaching aids, including the inclusion of information computer technologies in classes in many disciplines [8].

In the process of training, the future railway engineer of the specialization «Freight and commercial work, Mainline transport», the implementation of interdisciplinary must fully meet the requirements of the Federal State Educational Standard of Higher Education in the specialty being mastered. The success of a modern specialist depends not only on knowledge, skills and abilities, but also on the views, values, feelings, hopes, motivation, independence, cooperation, hard work and intuition of people. [9].

A university graduate should be able to apply the knowledge, skills and abilities acquired in the course of training in everyday and changing situations at work, demonstrating their competence [10].

Scientists consider interdisciplinary communication as one of the means of an integrated approach to teaching and upbringing. In modern pedagogy, an unambiguous definition of the concept of «interdisciplinary connections» does not exist, since this phenomenon is multidimensional and is not limited to the framework of the content, methods, forms of organization of education [11].

Interdisciplinary communication is a pedagogical condition that contributes to the reflection in the educational process of the formation of a holistic worldview that corresponds to the modern level of development of science and social practice, as well as mastering the skills of cognitive, educational, research and project activities by students. As a result, knowledge becomes not only specific, but also generalized, which gives students the opportunity to transfer this knowledge to new situations and apply it in practice [12].

Interdisciplinary connections in the training of students of higher educational institutions [13] are a concrete expression of the integration processes taking place today in science and in the life of society. Such connections play a special role in improving the practical and scientific-theoretical training of students, the main feature of which is the mastery of the generalized nature of students ' cognitive activity.

The study of all subjects of the natural science cycle is associated with mathematics. Mathematics provides students with a system of knowledge and skills necessary in the daily life of a person, forms general subject calculation and measurement skills. Successive links with the courses of the natural science cycle reveal the practical application of the mathematical knowledge and skills obtained by students, which contributes to the formation of a scientific worldview in students, ideas about mathematical modeling as a generalized method of understanding the world.

The use of joint knowledge from various subject areas of science is a regularity of modern competitive production that solves complex technical problems. A creative approach to any activity of a competent specialist is based on the ability to jointly apply knowledge and transfer ideas and methods from one area to another. The acquisition of such skills by a competitive specialist is the main social task of the university, which is solved with the help of interdisciplinary connections.

When using a subject-oriented learning system, interdisciplinary connections allow us to resolve the existing contradictions between the disparate assimilation of knowledge and the need for their application, both holistic and complex, in the practical life of a person.

The organization of an interdisciplinary approach at a university can be divided into two levels:

- Organization within a specific specialty;

- Organization within a certain block of academic disciplines.

When organizing the first level of interdisciplinary education, the main task will be to identify the sequence of studying the disciplines of the specialty. 
In addition, when organizing a second-level interdisciplinary approach, it is necessary to take into account:

- Correspondence of the content and forms of pedagogical influences to the real educational (information) capabilities of the trainees;

- ensuring the strength (stability) of the assimilation of educational material and the possibility of using the acquired knowledge, skills and abilities in accordance with the qualification characteristics of a specialist;

- ensuring the level of knowledge within the established time for studying the disciplines of the block in accordance with the curriculum.

The formation of students' knowledge and skills of using scientific content in the process of solving professional problems from any subject area is the main goal of mastering the discipline. For the successful implementation of professional activities, a young specialist must have a certain set of competencies that characterize him as a person and a professional and allow him to navigate his profession, be competitive in the labor market, and be ready for independent work [14].

Interdisciplinary connections are an important tool for the applied orientation of teaching mathematics. The possibility of using such relationships is explained by the fact that concepts of the same name are studied in mathematics and related disciplines [15], and mathematical means of expressing relationships between quantities are used in the study of related disciplines. Such mutual assimilation of knowledge and methods in various academic subjects has not only applied significance, but also creates favorable conditions for the formation of a scientific worldview.

Interdisciplinary communication can be implemented in a variety of ways. A more efficient way to achieve this goal is through applied problems from related disciplines, which allow students to demonstrate the use of mathematical methods to solve problems from other subject areas.

There are three types of interdisciplinary temporal relationships that the teacher has to deal with during teaching: antecedent, concomitant, and prospective.

Previous interdisciplinary connections include connections in the study of the course material, it is necessary to rely on previously acquired knowledge in other subjects.

Kinship interdisciplinary relationships are used when it is necessary to take into account that certain issues and definitions of certain concepts are studied both in mathematical modeling and in other subjects.

Among the main teaching methods used in the implementation of interdisciplinary relations, we have identified such as solving professionally oriented problems and organizing forms of education using the integration of mathematics and special disciplines.

\section{Results and discussion}

Mathematics is one of the few disciplines, each section of which allows you to perform certain calculations for the applied sciences and get certain conclusions.

In the implementation of interdisciplinary relations, in the conditions of the formation of a professional orientation, it is possible to use various forms of organizing a student's work, for example, the compilation and solution of problems with a production orientation. In problems of an applied nature, the problems under consideration must be solved using a mathematical apparatus. Applied tasks represent real conditions and situations that need to be solved in future professional activities [16]. Applied problems usually cover many areas of mathematics needed to study and analyze a specific situation. The professional orientation of teaching mathematics completely depends on the specific direction of training; therefore, it is necessary to carefully select problems of an applied nature, taking into account the interdisciplinary connections of the disciplines being taught. 
Therefore, for example, within the framework of the study of the discipline "Mathematics", in the second year of study, such a section of the discipline as Theory of Probability and the Foundations of Mathematical Statistics is studied.

Therefore, for example, in the framework of studying the discipline "Mathematics", in the second year of study, such a section of the discipline as Probability Theory and the Basics of Mathematical Statistics is studied. In the first group, students studied the material of the discipline using standard tasks not related to professional activity. Let us look at some of them.

Task 1.Three shooters, under the same and independent conditions, fired one shot at the same target. The probability of hitting the target by the first shooter is 0.9 , the second 0.8 , the third -0.7 . Find the probability that: a) only one of the shooters hit the target; b) at least two shooters hit the target.

Task 2.The probability that the required part is in the first, second, and third boxes, respectively, is $0.7,0,5,0,9$. Find the probability that the part is in only two boxes.

Task 3 . The probability of hitting the target with one shot is 0.9 . Find the probability that with 200 shots, the target will be hit at least 175 times.

Task 4. The probability of occurrence of an event in each of the independent tests is 0.2 . Find the probability that in 100 independent tests the event will occur 35 times.

Task 5. When harvesting potatoes with a potato digger, $10 \%$ of the potatoes are damaged. 4 potato tubers were selected at random. Write a binomial law of distribution of a discrete random variable $\mathrm{X}$ - the number of damaged potatoes among 4 selected ones and construct a polygon of the resulting distribution.

Task 6. The average length of the leaves of the garden currant in a certain area is $6.5 \mathrm{~cm}$. Individual deviations from this value are random, distributed normally with a mean square deviation of $1 \mathrm{~cm}$. One sheet is taken at random. Find the probability that its length deviates from the midline by no more than $0.5 \mathrm{~g}$.

Task 7. The spinner serves 1000 spindles. The probability of thread breakage on one spindle within $1 \mathrm{~min}$ is 0.004 . Find the probability that within 1 min breakage will occur on five spindles.

Task 8 . The batch contains $10 \%$ of non-standard parts. Four parts are selected at random. Write a binomial law of distribution of a discrete random variable X-the number of nonstandard parts among the four selected ones and construct a polygon of the resulting distribution.

Task 9. The probability of success in each trial is 0.4 . Find the probability that at 550 trials it will occur exactly 200 times.

The second group studied the section of the discipline with applied problems of engineering and economic sense, in solving which, firstly, interdisciplinary connections are realized, and secondly, students ' interest in the subject increases. Indeed, today, students' interest in the subjects of the natural science cycle is very low, and the teacher has to be interested in various tasks of a situational nature.

Let us consider some tasks.

Task 1. Oscillations of the time of movement of a train along the track are characterized by a normal distribution law with a mathematical expectation of a $=16$ minutes, and standard deviation 4 min. Determine the probability of a train running in more than 19 minutes.

Because it is necessary to find the probability of a train running in more than 19 minutes, then the solution of this problem is reduced to a solution by the formula:

$$
P(\alpha \leq X \leq \beta)=1-\left(F\left(\frac{\alpha-a}{\sigma}\right)-F\left(\frac{\beta-a}{\sigma}\right)\right)
$$


Task 2 . Of the 30 pneumatic drainage valves received by the assembly shop of the metro cars, 29 were checked. Three valves selected at random are installed on the object. What is the probability that all three valves have passed the test?

To solve this problem, we need to use the classical probability formula:

$$
P(A)=\frac{C_{29}^{3}}{C_{30}^{3}}=\frac{3659}{4060}=0.9
$$

Task 3. The repair depot should receive eight cars, of which two with damaged sides. By the specified time, 6 cars were received. What is the probability that not a single car with damaged sides was received?

To solve this problem, we need to use the classical probability formula:

$$
P(A)=\frac{C_{6}^{6}}{C_{8}^{6}}=\frac{1}{28}=0.036
$$

Task 4. Nine trains should arrive at the port, three of them with special equipment. It was reported that due to weather conditions, three trains would arrive one day late. What is the probability that trains with special equipment will be delayed?

Task 5. The probability of timely arrival at the destination station of a passenger train driven by a driver of a certain qualification is 0.9 . What is the probability that with a fivetime trip there will be three delays?[17]

Task 6. In the repaired radio station unit of the locomotive, the installer replaces two identical boards. The technical conditions are violated if both of them are with a reduced characteristic. The radio installer has 10 boards, of which three are with a reduced characteristic. Determine the probability of a violation of the technical specification with a random selection of two boards.

Task 7. On the way to the sorting park, when following the slide, unhook five switches. He can stop at each of them because of the resistance to movement with probabilities: at the first $p_{1}=0,2$, at the second $p_{2}=0,1$, at the third $p_{3}=0,15$, at the fourth $p_{4}=0,3$, and at the fifth $p_{5}=0,25$. Construct the law of distribution of the number of switches passed by the uncoupling to the stop. What is the most likely number of detachable switches? How many crossed switches can we mathematically expect for some average uncoupling?

Task 8 . When forming a railway train during the repair time, 22 cars have time to undergo high-quality repairs. To form a railway train, it is necessary that 20 cars undergo high-quality repairs. Cars that have undergone high-quality repairs are sent for repair again, independently of each other. The probability of poor-quality repairs is 0.1 . Find the probability of forming a railway train in one repair cycle.

Task 9. A batch of 120 manufactured wheelsets intended for the construction of special cars passes control and is then sent to a car-building plant. To receive a batch, it is necessary that three wheel pairs selected at random from the batch pass the control. What is the probability that the commission will accept a batch containing four faulty wheelsets?

\section{Conclusions}

In the methodology of teaching mathematics, one of the traditional directions is the problems of applied significance. As shown by pedagogical practice and research conducted in this area, when using problems of applied significance, the mathematical training of students in the system of vocational training is improved. One of the motives that stimulate interest in 
studying a particular question of the mathematics course is its practical significance, its connection with the future profession.

The results obtained at the stage of the final control of the assessment of knowledge shown in the diagram (Fig. 1.) show that when solving applied problems, students ' interest in the discipline of Mathematics increases by $80 \%$. When solving applied problems, the assimilation of the material increases, the percentage of passing the exam for the assessment «well» $(43 \%)$ and «excellent» $(37 \%)$ increases in the group. Students, solving such problems, do not have questions «Why do I study mathematics?»

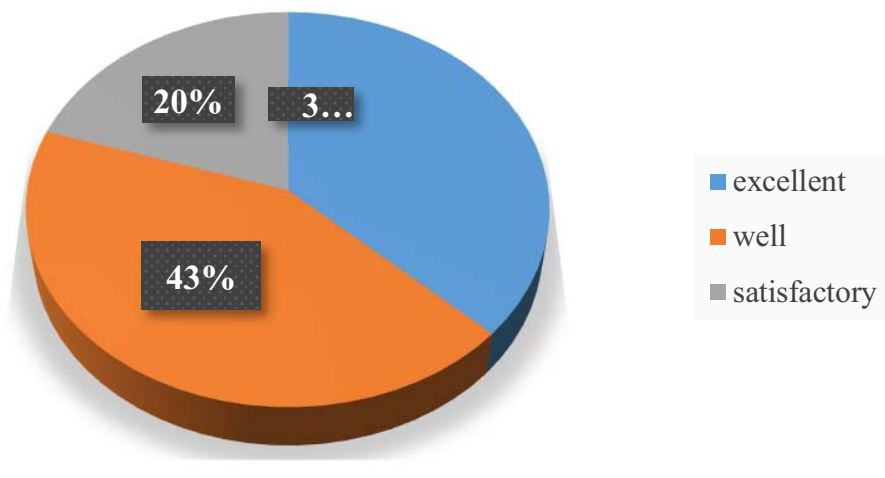

Fig. 1. The results of the second experimental group.

The results of the first group (Fig. 2), in which the material was studied on standard tasks without an applied orientation, are significantly worse (unsatisfactory (9\%), satisfactory $(56 \%))$, in comparison with the second group.

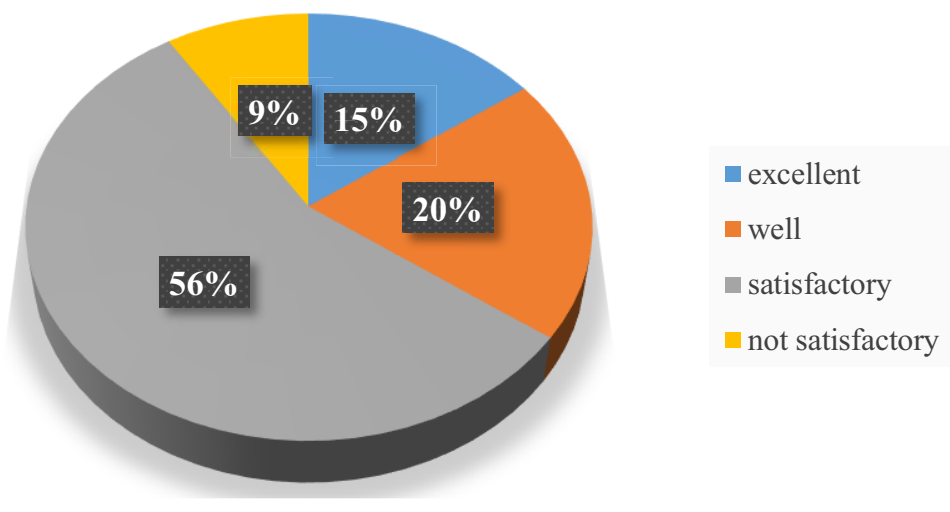

Fig. 2. The results of the first experimental group.

Thus, thanks to applied problems, we develop the connection of mathematics with special disciplines, which is not a little important when implementing new FSES in the 3 ++ generation. At such classes in mathematics, the necessary preparation of young people for constant "educational" activity is provided, and a readiness for self-education and selfdevelopment is formed. Under the influence of systematic interdisciplinary connections, general subject skills formed on different educational materials acquire the character of interdisciplinary skills. To date, any modern research involves the use of mathematical 
research methods, whether it is the processing and analysis of experimental results, or the construction of scientific theories [18].

\section{References}

1. J.A. Kamensky, Interdisciplinary connections in the learning process («Knowledge», M., 1955)

2. M. Vinogradova, N. Malchukova, S. Dorofeev, Journal of Physics: Conference Series 201 (2019) DOI: 10.1088/1742-6596/1333/7/072028

3. S.V. Kulikova, Problems of formation of value reference points in the education of rural youth. Collection of materials of the International scientific-practical conference, 99103 (2014)

4. N.V. Biryukova, Espacios 39(20), 4 (2018)

5. N. Biryukova, E3S Web of Conferences 222,5013 (2020) DOI: 10.1051/e3sconf/202022205013

6. M.V. Vinogradova, L.I. Yakobyuk, N.V. Zenina, Espacios 39(30) (2018)

7. L.I. Iakobiuk, M.V. Vinogradova, N.N. Malchukova, Y.V. Kryucheva, Espacios 38(40), 17 (2017)

8. L.I. Yakobiuk, The world of science culture and education 5(84), 179-181 (2020) DOI: 10.24411/1991-5497-2020-00923

9. M.V. Vinogradova, World of science, culture, education 3(82), 29-30 (2020) DOI: 10.24411/1991-5497-2020-00444

10. L.I. Iakobiuk, World of science, culture, education 6(79), 187-189 (2019) DOI: 10.24411/1991-5497-2019-10082

11. N.D. Anikeeva, Modern science-agro-industrial production collection of materials of scientific and practical international conference dedicated to the 135th anniversary of the first secondary school of the TRANS - Urals-Alexander real school and the 55th anniversary of the Northern TRANS-Ural state University (2014)

12. T.L. Blinova, A.S. Kirilova, Teaching skills: materials of the III mezhdunar. Science. Conf. (Buki-Vedi, Moscow, 2013)

13. I.V. Tolstoukhova, Y.V. Kryucheva, L.I. Iakobiuk, S.V. Kulikova, Humanities and Social Sciences Reviews 7(4), 899-905 (2019) DOI: 10.18510/hssr.2019.74120

14. N.N. Malchukova, Azimuth of scientific research 3(28), 161-164 (2019) DOI 10/26140/anip-2019-0803-0040

15. Y.V. Kryucheva, I.V. Tolstoukhova, L.I. Iakobiuk, M.V. Vinogradova, International Journal of Engineering and Technology(UAE) 7(4), 292-295 (2018) DOI: 10.14419/ijet.v7i4.38.24487

16. N.N. Malchukova, M.V. Vinogradova, I.E. Shemyakina, V.M. Breginya, World of science, culture, education 2(75), 109-111 (2019)

17. P.V. Gerasimenko, R.S. Kudarov, Theory of probability and mathematical statistics. Part 1. Theory of probability: collection of tasks (St. Petersburg State University of Railway Transport, St. Petersburg, 2013)

18. N.V. Biryukova, The world of science, culture, and education 6(85), 140-143 (2020) DOI: $10.24412 / 1991-5500-2020-685-140-143$ 